\title{
On-center/inhibitory-surround decorrelation via intraglomerular inhibition in the olfactory bulb glomerular layer
}

\author{
Thomas A. Cleland ${ }^{1,2}$ and Christiane Linster ${ }^{1,3}$ * \\ ${ }^{1}$ Computational Physiology Laboratory, Cornell University, Ithaca, NY, USA \\ ${ }^{2}$ Department of Psychology, Cornell University, Ithaca, NY, USA \\ ${ }^{3}$ Department of Neurobiology and Behavior, Cornell University, Ithaca, NY, USA
}

\section{Edited by:}

Alan Carleton, University of Geneva, Switzerland

Reviewed by:

Marcelo O. Magnasco, Rockefeller University, USA

Gilles Laurent, Max Planck Institute for Brain Research, Germany

*Correspondence:

Christiane Linster, Department of Neurobiology and Behavior, Cornell University, Ithaca, NY 14853, USA e-mail:cl243@cornell.edu
Classical lateral inhibition, which relies on spatially ordered neural representations of physical stimuli, cannot decorrelate sensory representations in which stimulus properties are represented non-topographically. Recent theoretical and experimental studies indicate that such a non-topographical representation of olfactory stimuli predominates in olfactory bulb, thereby refuting the classical view that olfactory decorrelation is mediated by lateral inhibition comparable to that in the retina. Questions persist, however, regarding how well non-topographical decorrelation models can replicate the inhibitory "surround" that has been observed experimentally (with respect to odor feature-similarity) in olfactory bulb principal neurons, analogous to the spatial inhibitory surround generated by lateral inhibition in retina. Using two contrasting scenarios of stimulus representation - one "retinotopically" organized and one in which receptive fields are unpredictably distributed as they are in olfactory bulb - we here show that intracolumnar inhibitory interactions between local interneurons and principal neurons successfully decorrelate similar sensory representations irrespective of the scenario of representation. In contrast, lateral inhibitory interactions between these same neurons in neighboring columns are only able to effectively decorrelate topographically organized representations. While anatomical substrates superficially consistent with both types of inhibition exist in olfactory bulb, of the two only local intraglomerular inhibition suffices to mediate olfactory decorrelation.

Keywords: olfactory bulb, decorrelation, non-topographical contrast enhancement, inhibition, computational model

\section{INTRODUCTION}

Complex sensory stimuli are represented by the concerted activation of populations of sensory neurons, each sensitive to a stimulus feature or group of features that constitute that neuron's receptive field. Highly similar stimuli consequently have correspondingly overlapping sensory representations in the brain - particularly in relatively peripheral sensory regions in which neuronal receptive fields are reasonably straightforward to measure. Pattern decorrelation is a common early stage transformation performed on the neural representations of sensory stimuli in multiple modalities that serves to reduce the overlap of similar sensory representations in a regulated manner, e.g., to improve the perceptual differentiation of physically similar stimuli (Joublin et al., 1996; Cook and McReynolds, 1998b; Wiemer et al., 2000; Mandairon et al., 2006; Mandairon and Linster, 2009). In particular, the oncenter/inhibitory surround ("Mexican-hat") transformation, also referred to as contrast enhancement, is a powerful form of pattern decorrelation characterized by a prominent inhibitory band in which relatively weakly activated neurons in the shoulder regions of a representation are actively and selectively inhibited below baseline levels (Figures 1Ai,ii). On-center/inhibitory surround response profiles have been observed in the in the early visual and auditory systems as well as in the olfactory system - although in the latter the surround is mapped with respect to a metric of odorant feature-similarity (Yokoi et al., 1995; Cleland, 2010).

In many primary and secondary sensory structures, neural responses form physically ordered representations of sensory stimulus attributes, mapping them in the brain with respect to the similarity of their features such that neurons with similar receptive fields are located correspondingly closely to one another. That is, physical proximity becomes a proxy for receptive field similarity. The neural mechanisms mediating similarity-dependent computations in these sensory modalities consequently are able to rely on short-range, lateral interconnections between neighboring neurons in order to preferentially couple neurons with the most highly overlapping receptive fields. Specifically, retinotopic, tonotopic, and somatosensory decorrelation can be mediated by nearest-neighbor lateral inhibitory interactions (von Bekesy, 1967; Yang et al., 1992; Suga et al., 1997; Cook and McReynolds, 1998a; Lavallee and Deschenes, 2004); each of these systems utilizes the resulting mutual inhibition among neurons with overlapping receptive fields to accentuate the differences between similar representations. Second-order principal neurons in the olfactory system, known as mitral cells, also exhibit on-center/inhibitory 
Ai

$$
\begin{aligned}
& \text { Input } \\
& \text { representations }
\end{aligned}
$$

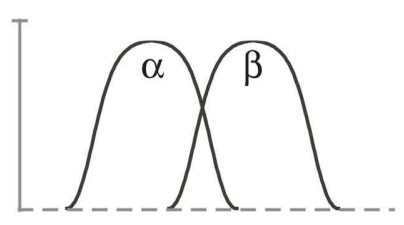

Aii

On-center/inhibitorysurround decorrelation

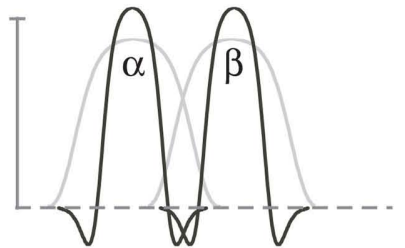

Aiii

$$
\text { Nonspecific (trivial) }
$$
decorrelation

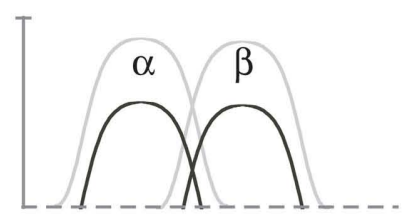

Bi

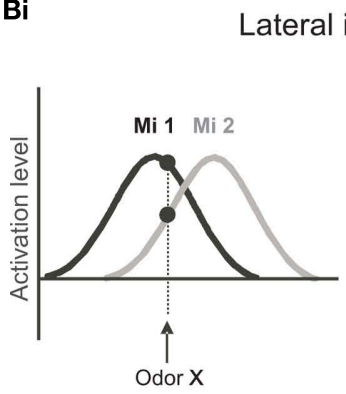

Bii

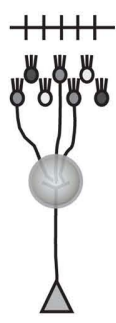

$\mathrm{HH}$
WW1⿴囗冂一 OSN

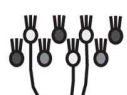

(1)

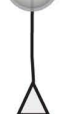

HWWWH Mi

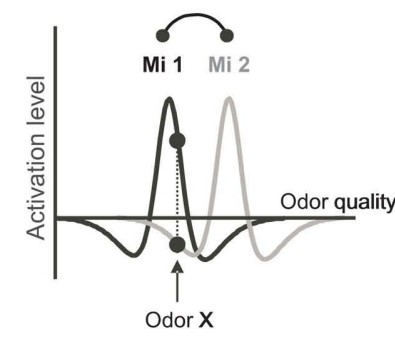

Biii

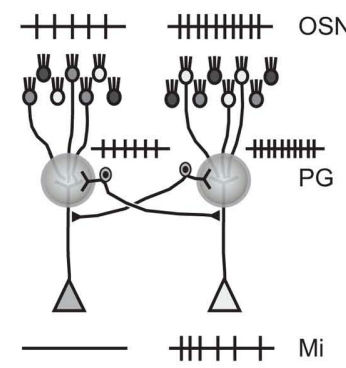

Ci
Intraglomerular inhibition

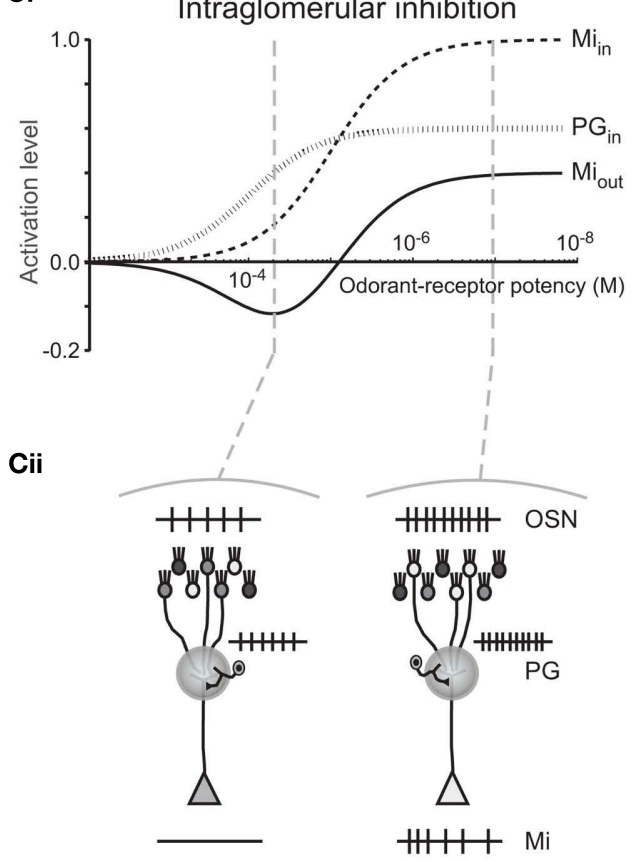

FIGURE 1 | Comparison of decorrelation models. (A) Schematic comparison of on-center/inhibitory surround and non-specific decorrelation functions. (i) Two overlapping input representations ( $\alpha$ and $\beta$ ) depicted in one dimension. (ii) Canonical on-center/inhibitory-surround decorrelation generates an explicit inhibitory surround in which the shoulders of the input representation are inhibited below baseline, yielding a sharp reduction in overlap among similar representations. This computation is performed by lateral inhibition in the retina and cochlear nucleus, and by the non-topographical model of olfactory receptive field decorrelation. (iii) $A$ lesser degree of decorrelation can be obtained by broad, non-specific inhibition, including lateral inhibition with an unstructured surround, although this imposes a general reduction in sensitivity across the entire representation. This is the effect of most lateral inhibitory models studied to date in the olfactory bulb; notably, it does not generate the inhibitory surround observed by Yokoi et al. (1995). Whereas both computations can effect a measurable decorrelation in principle, the two transformations differ both qualitatively and in terms of quantitative efficacy. Figure adapted from Cleland (2010). (B) Lateral inhibition. (i) Left panel. Tuning curves for two mitral cells (Mi 1 and $\mathrm{Mi}$ 2) with overlapping receptive fields for odorants, prior to the effects of lateral inhibition in a topographical representation scenario. Both neurons are excited by the odorant presented, although $\mathrm{Mi} 1$ is more strongly activated than Mi 2. Right panel. The same two mitral cell tuning curves after the inclusion of lateral inhibition. Now, whereas Mi 1 is still excited by the odorant presented, Mi 2 is inhibited. The abscissa is a hypothetical axis of odor quality. (ii) Schematic representation of neuronal responses to a given odorant in the absence of lateral inhibitory PG axonal projections in a topographical representation scenario. The odorant presented activates the lightly shaded population of OSNs somewhat more strongly than it does the more darkly shaded population of OSNs, evoking a higher spike rate in the
OSN population projecting to the glomerulus on the right. In the absence of inhibition, mitral cells (Mi) are activated in direct proportion to their constituent OSN populations. (iii) Schematic representation of the same two glomeruli and the same odorant presented as in (Bii), with the addition of PG cells that also are activated in direct proportion to their OSN population and deliver lateral inhibition onto mitral cells in the other glomerulus. The mitral cell that is more weakly responsive to the odorant presented [corresponding to the dotted vertical line in $\mathbf{( B i})$ ] is silenced due to this lateral inhibitory input from the PG cell associated with the more strongly activated parent glomerulus. (C) Intraglomerular inhibition. (i) Tuning curves for mitral and periglomerular cells mapped onto an abscissa of odor ligand-receptor potency (potency incorporates both ligand-receptor affinity and efficacy terms; for discussion of the effects of odor concentration on this relationship, see Cleland et al., 2007). Both mitral and periglomerular cells are excited by the odorant presented via the activity of their associated OSN populations $\left(\mathrm{Mi}_{\mathrm{in}}, \mathrm{PG}_{\mathrm{in}}\right)$; though $\mathrm{PG}$ cells are more sensitive to this common input (Gire and Schoppa, 2009). Inhibition of mitral cells by PG cells alters the mitral cell tuning curve ( $\mathrm{Mi}_{\text {out }}$ ), generating a Mexican-hat inhibitory surround in a metric space defined by odor quality. Figure adapted from Cleland and Sethupathy, 2006). (ii) Schematic representation of neuronal responses to a given odorant in the presence of intraglomerular PG-mediated inhibition of mitral cells. The odorant presented is the same as in (B), exhibiting a stronger potency for the receptors expressed by the OSN population projecting to the glomerulus on the right [the two potencies correspond to those depicted by vertical lines in (Ci)]. Periglomerular cells are activated in direct proportion to their constituent OSN populations, as in the lateral inhibitory case, whereas mitral cells receive both afferent excitation and intraglomerular inhibition, thereby exhibiting sharpened receptive fields with inhibitory surrounds. The mitral cell that is more weakly responsive to the odorant presented is silenced [compare to (Biii)]. 
surround response properties that clearly reflect decorrelation among structurally and perceptually similar odor stimuli (Yokoi et al., 1995). However, in the olfactory bulb - the layered telencephalic structure within which mitral cells are embedded - it recently has been demonstrated that no comparably orderly topographical mapping of odor stimulus similarities exists (Soucy et al., 2009), a result previously predicted on theoretical grounds (Cleland and Sethupathy, 2006; Cleland et al., 2007). Consequently, a nearest-neighbor lateral inhibition mechanism is fundamentally unable to compute the observed decorrelations among stimuli of similar quality in the olfactory bulb as it does in some other sensory structures. In contrast, purely local computations, utilizing only established and well-described synaptic interactions in the olfactory bulb glomerular layer, are able to perform olfactory stimulus decorrelation in a manner that is completely independent of the topographical mapping of odorant features and hence is able to perform the necessary transformations irrespective of any basis for the physical mapping of the odor representation (Cleland and Sethupathy, 2006; Cleland et al., 2007; Cleland, 2010). We here compare these two on-center/inhibitory-surround mechanisms directly, using otherwise-identical computational models, in order to demonstrate (1) their equivalence when presented with topographically organized, low-dimensional inputs and (2) the capacity of the non-topographical contrast enhancement (NTCE) algorithm to perform this same computation on highdimensional, distributed, or disorganized representations within which topographical lateral inhibition fails.

Additional models of olfactory decorrelation also have been proposed (see Discussion). Most of these reduce to a form of non-specific decorrelation that does not map effectively onto odorant feature-similarity; these lack an inhibitory surround and instead achieve a measure of decorrelation simply by reducing general sensitivity (Figure 1Aiii). The exceptions are learning-based models that construct experience-dependent maps of inhibitory efficacy by virtue of statistical learning (e.g., Cecchi et al., 2001). These models are based on feature-covariance rather than featuresimilarity per se, and are likely to follow, rather than replace, early stage mechanisms based on features of physical similarity (see Discussion). The present analysis is restricted to the mechanisms underlying the initial decorrelation of representations based upon their physical similarities.

We constructed full-scale neural circuit models of the olfactory bulb glomerular layer and implemented two alternative mechanisms proposed to mediate decorrelation in the olfactory bulb glomerular layer. The first (lateral inhibition) relies on nearestneighbor lateral inhibitory projections that are excited by afferent input and inhibit olfactory bulb principal neurons (mitral cells) in their immediate neighborhood (Pinching and Powell, 1971, 1972; Linster and Gervais, 1996; Linster and Hasselmo, 1997; Figure 1B). This lateral inhibitory mechanism resembles that of the retina and cochlear nucleus, and is able to perform contrast enhancement in models of the olfactory bulb (or insect antennal lobe) provided that olfactory receptive field similarities are systematically ordered in a one- or two-dimensional map (Linster and Hasselmo, 1997; Linster and Cleland, 2004). The second mechanism (intraglomerular inhibition) relies on local inhibitory interactions between periglomerular cells and mitral cell dendrites within single glomeruli, via a location-independent algorithm (Cleland and Sethupathy, 2006; Cleland et al., 2007; Figure 1C). We then tested each of these mechanisms against two scenarios of bulbar odor representation: one in which physically neighboring glomeruli were activated by receptors responding to correspondingly similar odorant features (i.e., a retina-like topographical representation), and one in which the same set of glomerular responses was randomly disbursed throughout the glomerular layer (a distributed representation, as has been demonstrated in olfactory bulb; Soucy et al., 2009). Our simulations showed that in the case of topographical odor representations, both mechanisms perform comparably well and generate similar decorrelated output patterns, whereas only the intraglomerular inhibition mechanism can successfully decorrelate distributed odor representations. We propose that the intraglomerular inhibition mechanism is the initial mediator of stimulus decorrelation within the olfactory bulb in that it relies upon an experimentally established synaptic architecture in the glomerular layer, is independent of the physical mapping of odor representations, and in particular does not depend on a non-existent retina-like topographical representation of feature similarity.

\section{MATERIALS AND METHODS}

\section{GLOMERULAR NETWORK ARCHITECTURE}

Primary olfactory sensory neurons (OSNs) in the nasal epithelium project axons into the olfactory bulb. Canonically, the axons of OSN populations that express the same type of odorant receptor converge to form glomeruli on the surface of the olfactory bulb such that the odor response profile of each glomerulus directly reflects the receptive field of its constituent OSNs. Within these glomeruli, the axonal arbors of the convergent OSN populations synaptically excite the dendritic arbors of second-order principal neurons (e.g., mitral cells) and inhibitory interneurons (e.g., periglomerular cells). Periglomerular cells in turn inhibit the primary dendrites of mitral cells within the same glomerulus, and some also project axons to neighboring glomeruli.

\section{SIMULATION PARAMETERS}

A total of 1024 glomeruli with associated OSNs, PG cells, and mitral cells were simulated, arranged in a two-dimensional $32 \times 32$ torus (i.e., the edges of the map wrap around independently). In comparison, the olfactory bulbs of rats and mice contain 1000-1200 chemotopically distinct glomeruli corresponding to the 1000-1200 different olfactory receptors expressed by these animals (as most glomeruli are duplicated in the medial and lateral $\mathrm{OB}$, the actual counts of anatomical glomeruli in each olfactory bulb are roughly twice this number). Synaptic connections and parameter values are presented in Table 1. The pattern of projections from PG cells to mitral cells in the model was structured to produce either lateral (interglomerular) inhibition with projections extending up to five glomerular diameters (Figure 1Biii) or local (intraglomerular) inhibition in which lateral projections were omitted entirely (Figure 1Cii). These constituted the only variable parameters in the present simulations.

For simplicity, concentration was not substantially varied, so that the bulbar circuitry mediating intensity compression and partial normalization observed in mitral cell activity profiles could be 
Table 1 | Computational model parameters

\begin{tabular}{ll}
\hline $\begin{array}{l}\text { Olfactory sensory } \\
\text { neurons (OSN) }\end{array}$ & $\tau=2.0 \mathrm{~ms} ; \theta_{\min }=0.0 ; \theta_{\max }=1.0$ \\
$\begin{array}{l}\text { Periglomerular } \\
\text { cells (PG) }\end{array}$ & $\tau=2.0 \mathrm{~ms} ; \theta_{\min }=0.0 ; \theta_{\max }=2.0$ \\
$\begin{array}{l}\text { Mitral cells (Mi) } \\
\text { Afferent, OSN }\end{array}$ & $\tau=10.0 \mathrm{~ms} ; \theta_{\min }=0.0 ; \theta_{\max }=6.0$ \\
to PG & $W_{\mathrm{OSN}-\mathrm{PG}}=0.014 ; E_{\mathrm{N}, \mathrm{OSN}-\mathrm{PG}}=+70 ; \tau_{1}=1.0 ; \tau_{2}=2.0$ \\
Afferent, OSN to Mi & $W_{\mathrm{OSN}-\mathrm{Mi}}=0.014 ; E_{\mathrm{N}, \mathrm{OSN}-\mathrm{Mi}}=+70 ; \tau_{1}=1.0 ; \tau_{2}=2.0$ \\
$\begin{array}{l}\text { Intraglomerular } \\
\text { inhibitory, PG to Mi }\end{array}$ & $W_{\mathrm{PG}-\mathrm{Mi}}=0.01 ; E_{\mathrm{N}, \mathrm{PG}-\mathrm{Mi}}=-5 ; \tau_{1}=4.0 ; \tau_{2}=8.0$ \\
Interglomerular & $W_{\mathrm{PG}-\mathrm{Mi}}=0.004 ; E_{\mathrm{N}, \mathrm{PG}-\mathrm{Mi}}=-5 ; \tau_{1}=4.0 ; \tau_{2}=8.0 ;$ \\
inhibitory, PG to Mi & \\
\hline
\end{tabular}

The instantaneous spiking probability for each cell type is a continuous, bounded function of the membrane potential with a threshold $\theta_{\min }$ and a saturation value $\theta_{\text {max }}$. Omega values $\left(w_{i j}\right)$ designate synaptic weights, and values of $E_{N, i j}$ designate synaptic reversal potentials. $\tau$ designates the membrane time constant, and $\tau_{1}$ and $\tau_{2}$ the synaptic time constants. All model parameters were chosen randomly $\pm 10 \%$ around the above mean values for each simulation.

omitted from the simulations. Both intraglomerular and lateral inhibitory mechanisms function only within limited ranges of afferent input intensity; compression of the range of OSN input intensities is likely regulated by presynaptic inhibition of OSN terminals (Pirez and Wachowiak, 2008; Cleland et al., 2012). Intraglomerular inhibition additionally requires a normalizing mechanism to establish competitive interactions among olfactory bulb columns; the existence of such competitive normalization is indicated by the non-monotonic response levels observed in mitral cells' responses to increasing odorant concentrations, and has been theoretically attributed to a bulbar lateral excitatory network comprising external tufted (ET) cells and superficial short-axon cells (Cleland et al., 2007; Cleland, 2010). Subsequent computations performed by deep bulbar circuitry (e.g., mitral cell interactions with granule cells; see Discussion), secondary cortices such as piriform cortex, and ascending feedback projections to the olfactory bulb also were omitted.

Simulated odorants each activated a characteristic subset of OSNs in the model. Specifically, each model OSN had a normally distributed receptive field (with variable SDs and amplitudes; see Table 1) with ligand-receptor potency drawn randomly from this distribution for each odorant. Using a topographical representation, OSNs projecting to neighboring glomeruli expressed similar and overlapping receptive fields (Figures $\mathbf{1 B i}$ and $2 \mathbf{A}$ ), such that the activation amplitude of each OSN was chosen as a function of its glomerular distance from the OSN centered on that odorant with the SD chosen randomly from a uniform distribution ranging from $\sigma=1.0$ to 5.0 glomerular diameters. In the case of distributed representations, the same statistical distribution of response profiles was randomly distributed across the $32 \times 32$ array (Figure 2B). The synaptic interactions between PG cells and mitral cells were either local (intraglomerular), such that each PG cell inhibited the mitral cell associated with the same glomerulus and receiving the same OSN input (Figure 1C), or lateral, such that each PG cell inhibited mitral cells associated with neighboring glomeruli located within a radius of five glomerular diameters surrounding the PG cell's glomerulus (Figure 1B). This radius was chosen to match the lengths of PG cell axons measured in the rat olfactory bulb (Pinching and Powell, 1972).

\section{MODEL NEURON EQUATIONS}

All neurons were represented as single compartments except for mitral cells, which were represented by four compartments (glomerular tuft, primary dendrite, secondary dendrite, and soma). Each compartment was characterized by a membrane time constant that can be regarded as the mean product of the membrane capacitance and the membrane input resistance. Consequently, the evolution of the membrane voltage over time is described by a first order differential equation:

$\tau \frac{\mathrm{dv}(t)}{\mathrm{dt}}+\mathrm{v}(t)=\mathbf{I}_{\mathrm{ext}}(t)$

where $\tau$ is the charging time constant of the neuron and $\mathrm{I}_{\text {ext }}(t)$ is the total input at time $t$. The input from a particular presynaptic neuron at time $t$ is computed as a function of the synaptic strength $w_{\mathrm{ij}}$, the conductance change $\mathrm{g}(t)$ due to a presynaptic event $\mathrm{x}_{j}$ at time $\mathrm{t}_{j}$ (either a unitary event representing an action potential or an analog value in case of a non-spiking neuron), and the difference between the Nernst potential $\mathrm{E}_{\mathrm{N}, \mathrm{ij}}$ of the associated channel type and the current membrane potential $\mathrm{v}_{\mathrm{i}}(t)$ of the postsynaptic neuron:

$\mathrm{I}_{\mathrm{i}, \mathrm{ext}}(t)=\sum_{\mathrm{j}} w_{\mathrm{ij}} \sum_{t \mathrm{j}<t} g\left(t-t_{\mathrm{j}}\right)\left[E_{\mathrm{N}, \mathrm{ij}}-\mathrm{v}_{\mathrm{i}}(t)\right]$.

The time course of $\mathrm{g}(t)$ is described by a double exponential function:

$\mathrm{g}\left(t-t_{\mathrm{j}}\right)=\mathrm{g}_{\max } \frac{\tau_{1} \tau_{2}}{\tau_{1}-\tau_{2}}\left(\mathrm{e}^{-\left(t-t_{\mathrm{j}}\right) / \tau_{1}}-\mathrm{e}^{-\left(t-t_{\mathrm{j}}\right) / \tau_{2}}\right)$

Mitral cell somatic compartments produced discrete spikes of unit amplitude for output, computed according to the instantaneous spiking probability, a continuous, bounded function of the membrane potential with a threshold $\theta_{\min }$ and a saturation value $\theta_{\max }$. (For a large number of neurons this translates to average spike rates being a quasi-linear function of the membrane potential). The continuous outputs of all other neurons were calculated from the same function. When networks were built, all parameters were chosen randomly $\pm 10 \%$ around the mean values indicated in Table 1 to ensure that the results were not based solely on a specific combination of parameters.

\section{DECORRELATION CALCULATIONS}

To calculate the overlap between representations and thereby measure the effectiveness of pattern decorrelation, 60 simulations, each using a new pair of randomly determined odors, were run for each of the four conditions (topographical/lateral, topographical/intraglomerular, distributed/lateral, distributed/intraglomerular). Each odorant was represented as a 1024-element activity vector in which each element represented the activity at one glomerulus; specifically, the average output activity of the corresponding OSN or mitral cell over the course of 

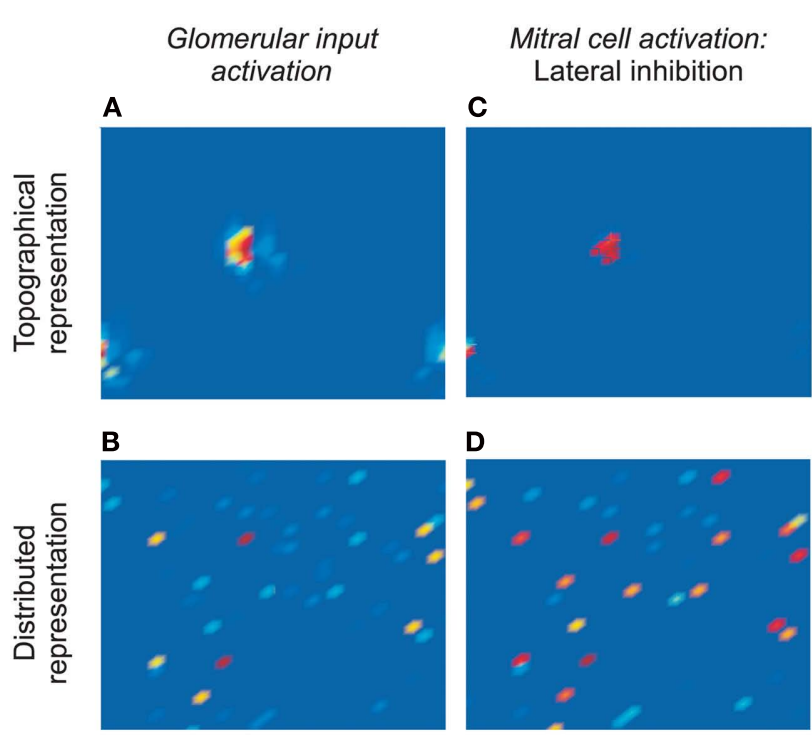

D

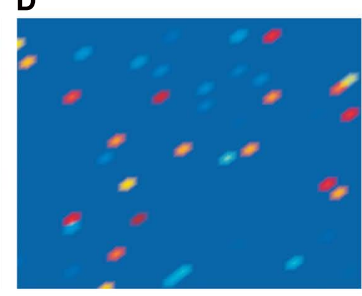

Mitral cell activation: Intraglomerular inhibition

E

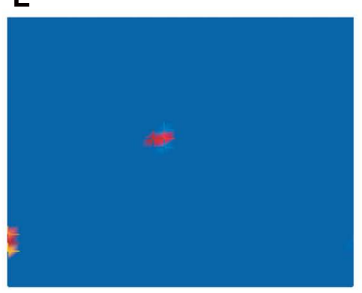

$\mathbf{F}$

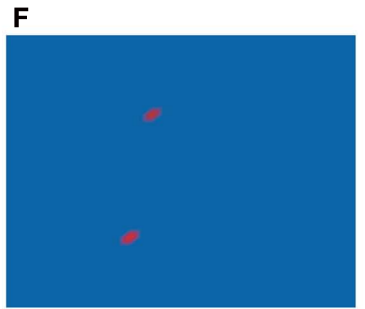

FIGURE 2 | Simulations of odor-evoked activity and the efficacy of decorrelation mechanisms. (A) Response of a $32 \times 32$ array of glomeruli to presentation of a model odorant, prior to the application of inhibition (corresponds to Figure $\mathbf{1 B i i}$, or to $\mathbf{M i}_{\text {in }}$ in Figure $\mathbf{1 C i}$ ). The locations of activated glomeruli were grouped into two clusters according to their levels of activation in order to replicate a retina-like topology of similarity (topographical representation; the lower right and lower left sub-clusters are contiguous because the map wraps around). (B) The same response profile as in (A), except that the locations of activated glomeruli were distributed randomly across the $32 \times 32$ matrix (distributed representation). Note that the deep red-colored glomeruli are the most strongly activated in both representations. (C) Response of the $32 \times 32$ array of mitral cells in the topographical representation scenario after the effects of lateral inhibition are incorporated. The mitral cell activation profile is sharper and narrower at each of the two clusters, such that only the most strongly activated mitral cells remain active. (D) Response of the $32 \times 32$ array of mitral cells in the distributed representation scenario after the effects of lateral inhibition are incorporated. The mitral cell activation profile is broad and disorganized, with an unclear relationship to the pre-inhibition representation. (E) Response of the $32 \times 32$ array of mitral cells in the topographical representation scenario after the effects of intraglomerular local inhibition are incorporated. The mitral cell activation profile is sharper and narrower at each of the two clusters, such that only the most strongly activated mitral cells remain active - replicating the effect of lateral inhibition in this scenario. (F) Response of the $32 \times 32$ array of mitral cells in the distributed representation scenario after the effects of intraglomerular local inhibition are incorporated. The mitral cell activation profile is sharper and narrower, such that only the most strongly activated mitral cells remain active. (C,E,F) depict effective decorrelation of the input representations. a 200-ms stimulation. The overlaps between the representations of each odor stimulus pair at both the input level (OSN activation) and the output level (mitral cell activation) were calculated as the normalized dot product between the corresponding 1024-element activity vectors $\mathrm{O} 1$ and $\mathrm{O} 2$ :

Overlap $_{\mathrm{O} 1-\mathrm{O} 2}=\frac{\sum_{i=1}^{N} \mathrm{o} 1_{\mathrm{i}} \mathrm{o} 2_{\mathrm{i}}}{\|\mathrm{O} 1\|\|\mathrm{O} 2\|}$

where $\mathrm{o} 1_{\mathrm{i}}, \mathrm{o} 2_{\mathrm{i}}$ are the elements of the activity vectors $\mathrm{O} 1$ and $\mathrm{O} 2$ (respectively) and $\|\mathrm{O} 1\|,\|\mathrm{O} 2\|$ are the norms of vectors $\mathrm{O} 1$ and $\mathrm{O} 2$. To quantify the degree of decorrelation produced by each of the four conditions, a decorrelation index was calculated for each odor pair as follows:

Decorrelation $_{\mathrm{O} 1-\mathrm{O} 2}=1-\frac{\text { mitral-overlap }_{\mathrm{O} 1-\mathrm{O} 2}}{\text { OSN-overlap }_{\mathrm{O} 1-\mathrm{O} 2}}$

where mitral-overlap and OSN-overlap are the overlaps between each odor stimulus pair at the output and input levels, respectively. For each of the four conditions, the 60 decorrelation indices then were averaged; the average then was subtracted from 1 and multiplied by 100 for reporting decorrelation under each condition as a percentage reduction in overlap.

\section{RESULTS}

We compared the respective capabilities of the lateral inhibition and intraglomerular inhibition mechanisms to decorrelate odor representations in the olfactory bulb glomerular layer based on two possible scenarios of glomerular organization. The first scenario, topographical representation, resembles the retina in that odor representations are fully ordered in a two-dimensional framework such that the physical proximity of glomeruli is predictive of the similarity of their response profiles (i.e., the overlap in their receptive fields). In the second, contrasting scenario of distributed representation, the odor response profiles of individual glomeruli are unrelated to their physical proximity, as observed in olfactory bulb (Soucy et al., 2009). Aside from the physical positioning of glomeruli, the distribution of glomerular responses was identical in both scenarios.

\section{LATERAL INHIBITION MODEL}

In the case of lateral inhibition, PG cells in the model receive afferent sensory input from primary OSNs within the single (parent) glomerulus to which that population of OSNs projects. 
PG cell axons projecting to neighboring glomeruli inhibit mitral cells therein that are activated by different populations of OSNs (Figure 1B). In the topographical representation scenario, PG cells would thereby inhibit mitral cells with highly similar receptive fields to those of their parent glomerulus, i.e., exhibiting activation curves that are adjacent or overlapping on an axis (or plane) of stimulus variation. As a result, all mitral cells are inhibited by a subset of the odorant features that activate their parent glomerulus; these features constitute the inhibitory surround of these cells' receptive fields (Figure 1Bi). Importantly, while in the topographical representation scenario this inhibitory-surround correlates with the spatial localization of glomeruli, it is the surround in odor feature-similarity space (Yokoi et al., 1995) that is actually relevant in the olfactory modality.

Figures 1Bii,iii depict a schematic representation of neuronal responses to a given odorant in the absence (Figure 1Bii) and presence (Figure 1Biii) of lateral inhibitory PG axonal projections in a topographical representation scenario. The mitral cell that is more weakly responsive to the odorant presented (vertical lines in Figure $1 \mathbf{B i}$ ) is silenced due to this lateral inhibitory input from the PG cell associated with the more strongly activated parent glomerulus. Consequently, the number of mitral cells activated by a given odorant stimulus is reduced, and the overlap between the neural representations of similar stimuli is reduced; i.e., the representations are decorrelated. In contrast, in the distributed representation scenario, the same distribution of lateral inhibitory PG projections would inhibit neighboring mitral cells exhibiting receptive fields that may be similar, relatively dissimilar, or entirely unrelated to those of the parent glomerulus. The pattern of lateral inhibition therefore is essentially random with respect to odor feature-similarity space, and hence fails to decorrelate odor representations except in the trivial sense that is achievable through uniform or disorganized inhibition (Figure 1Aiii).

\section{INTRAGLOMERULAR INHIBITION MODEL}

In the intraglomerular inhibition model, in contrast, PG cells only inhibit mitral cells that are associated with the same parent glomerulus; hence, the physical locations of glomeruli are irrelevant. Both mitral cells and PG cells are excited by afferent OSN activity in a manner identical to the lateral inhibition model. Because PG cells are intrinsically more responsive to afferent input than are their coglomerular mitral cells (Gire and Schoppa, 2009) presumably owing to their exceptionally high input resistance and the colocalization of OSN inputs and voltage-dependent GABA release mechanisms within the same dendritic gemmules - mitral cells begin to receive GABAergic shunt inhibition from PG cells at odor ligand-receptor potencies that are weaker than the potencies at which they will first receive sufficient direct excitation by OSNs to approach spike threshold (Cleland and Sethupathy, 2006; Figure 1Ci). Consequently, mitral cells are inhibited below baseline by a subset of the odorant features that activate their parent glomerulus - specifically, those features for which the potency of the odorant ligand for its OSN receptors is modest. These features constitute the inhibitory surround of the mitral cells' receptive fields. Importantly, this inhibitory surround is intrinsically based in the odor feature-similarity space that is relevant in the olfactory modality (Yokoi et al., 1995), irrespective of the spatial locations of glomeruli. Notably, when presented with a two-dimensional topographically organized representation that can be meaningfully decorrelated by lateral inhibition, both the lateral and the intraglomerular inhibition mechanisms produce similar on-center/inhibitory-surround transformations (compare bottom traces in Figures 1Biii,1Cii).

\section{COMPARISON OF LATERAL AND INTRAGLOMERULAR INHIBITION}

We then simulated the transformations effected by both mechanisms of decorrelation acting on both scenarios of odor representation (Figure 2). Specifically, we simulated 1024 OSN populations projecting to the same number of glomeruli arranged in a $32 \times 32$ array. Odor stimuli were modeled as distributions of odor ligandreceptor potencies such that a substantial minority of OSNs was activated to differing degrees by model odor stimulation. This distribution of potencies, and hence of glomerular response profiles, was kept constant in all simulations. However, in the topographical representation scenario, we arranged the activated glomeruli in spatially localized clusters, with the most strongly activated glomeruli in the centers of these clusters, simulating a twodimensional bulbar chemotopy (Figure 2A). In contrast, in the distributed representation scenario, the activated glomeruli were positioned randomly (Figure 2B).

The activity of model mitral cells associated with each of these glomeruli was recorded and mapped into a corresponding $32 \times 32$ array. In the absence of either form of inhibition, mitral cell activity patterns corresponded exactly to glomerular input activity patterns (Figures 2A,B). The addition of lateral or intraglomerular inhibition, however, transformed the odor representations in characteristic ways. Specifically, lateral inhibition successfully decorrelated topographical odor representations (Figure 2C), reducing the population of activated mitral cells to those that initially were most strongly activated (and therefore centrally located within clusters of activated glomeruli). However, lateral inhibition failed entirely to decorrelate distributed representations (Figure 2D). In contrast, the intraglomerular inhibition algorithm decorrelated both topographical and distributed representations successfully (Figures 2E,F); in both cases mitral cell representations were reduced to those associated with the most strongly activated glomeruli, irrespective of their spatial locations. Indeed, in the topographical representation case, the two mechanisms produced essentially the same result (compare Figures 2C,E).

To quantify the efficacy of decorrelation between the representations of similar odorants that could be achieved by each mechanism, we ran 60 simulations for each pairing of mechanism and representation (240 simulations total) in which the patterns of odor stimulation across the 1024 glomeruli were generated randomly for a pair of simulated odorants. For the odor pair in each such simulation, we calculated the overlap between the representations at the OSN/glomerular input level (i.e., before the effects of inhibition) as well as their overlap at the mitral cell level (after inhibition) by calculating the normalized dot product between the 1024-dimensional vectors spanned by the average output activities of each OSN/glomerulus or mitral cell during the course of a 200-ms odor presentation (see Methods). We then plotted the pairwise mitral cell overlaps against the corresponding 


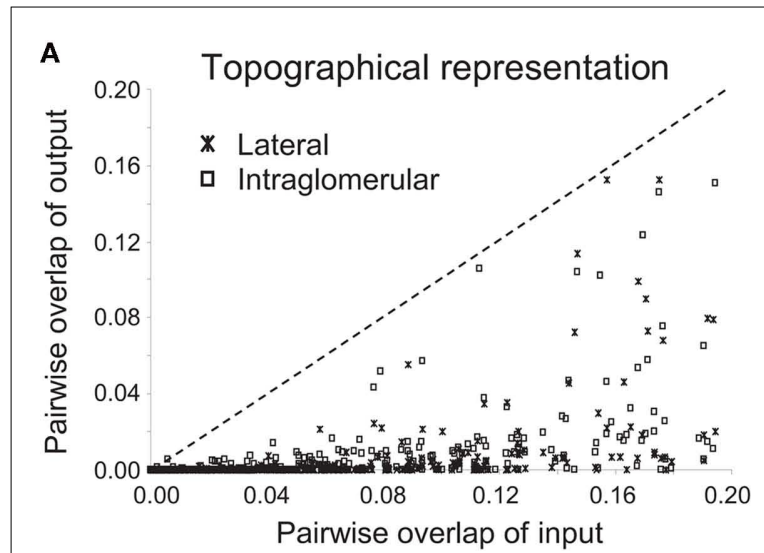

B

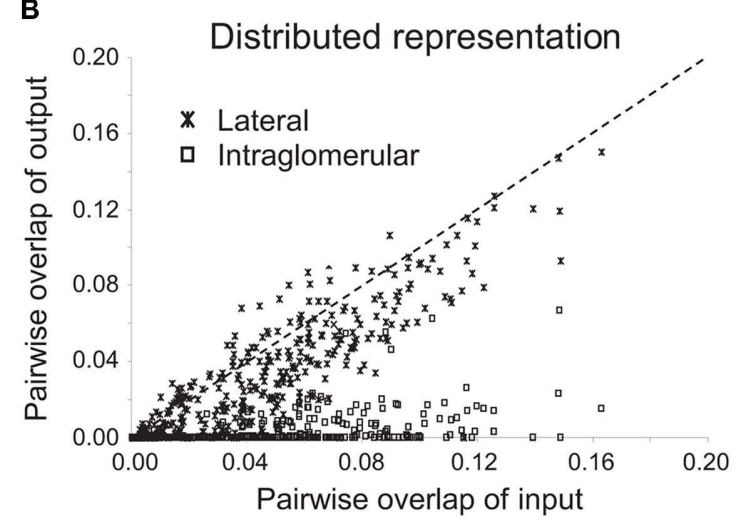

。

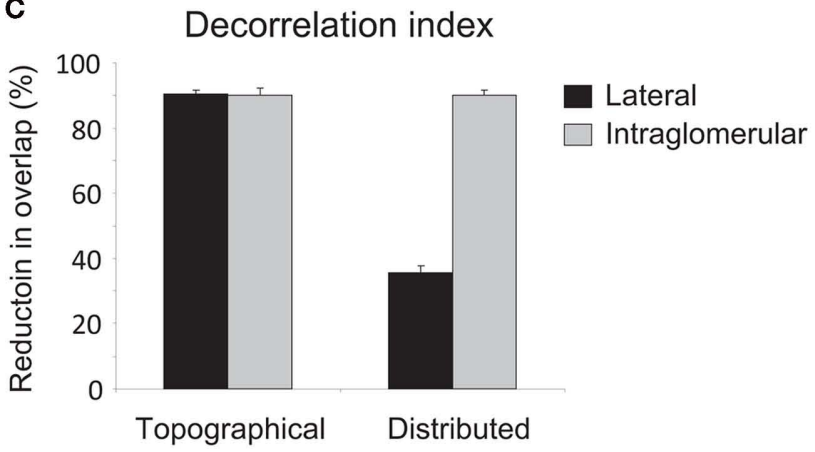

FIGURE 3 | Quantification of the efficacy of decorrelation mechanisms on different representation scenarios. (A) Efficacy of lateral and intraglomerular decorrelation mechanisms on topographical representations. Sixty simulations for each mechanism were run using randomized model odorant pairs with differing degrees of similarity. The overlap between each of these paired odorants prior to the application of inhibition (corresponding to Figures 2A, B) was then plotted against their overlap following lateral or intraglomerular inhibition (corresponding to Figures $\mathbf{2 C}, \mathbf{E}$, respectively). Points lying substantially below the diagonal signify effective decorrelation of the representations of those odorant pairs; points lying along the abscissa signify representations decorrelated to the extent that they no longer overlap at all. Both lateral and intraglomerular inhibition mechanisms effectively decorrelated topographical representations. (B) Efficacy of lateral and intraglomerular decorrelation mechanisms on distributed representations. Sixty simulations for each mechanism were run using randomized model odorant pairs with differing degrees of similarity. The overlap between each of these paired odorants prior to the application of inhibition (corresponding to Figures $\mathbf{2 A}, \mathbf{B}$ ) was

(Continued)

\section{FIGURE 3 | Continued}

then plotted against their overlap following lateral or intraglomerular inhibition (corresponding to Figures 2D,F, respectively). Points lying substantially below the diagonal signify effective decorrelation of the representations of those odorant pairs; points lying along the diagonal signify no decorrelation, whereas points above the diagonal indicate increased overlap (confusion) among representations. Only intraglomerular inhibition mechanisms effectively decorrelated distributed representations. (C) Decorrelation index of the proportional reduction in overlap between input and output representations in all four mechanism/representation cases. Both lateral and intraglomerular inhibitory mechanisms decorrelated topographical representations by up to $90 \%$; however, only intraglomerular inhibition successfully decorrelated similar distributed representations to this degree. Lateral inhibition was not an effective algorithm to decorrelate distributed representations, although even this disorganized inhibition produced a modest net decorrelation simply by silencing some neurons that would otherwise be active.

pairwise OSN overlaps (Figure 3; 1200 pairwise comparisons). In these plots, data points that lie along the diagonal indicate that no decorrelation between odor pairs has been achieved; i.e., the overlap at system output is the same as the overlap at system input). Data points that lie beneath the diagonal (dotted line) indicate decorrelation whereas values above the diagonal indicate the opposite of decorrelation (increased overlap between similar representations).

These simulations demonstrate that both lateral and intraglomerular inhibitory interactions can decorrelate topographical representations, reducing the overlap of the output (mitral cell activity profiles) as compared with the input (Figure 3A). However, only intraglomerular inhibition successfully decorrelated distributed representations; lateral inhibition, on average, had minor and inconsistent effects on the correlations of odor representations (Figures 2D and 3B; see below). Finally, we calculated a decorrelation index (the proportional reduction in overlap between input and output representations in all four cases; Figure 3C). Whereas both lateral and intraglomerular inhibitory mechanisms reduced the overlap of similar topographical representations by up to $90 \%$, only intraglomerular inhibition successfully decorrelated similar distributed representations to this degree. Lateral inhibition was not an effective algorithm to decorrelate distributed representations.

The roughly $35 \%$ decorrelation achieved by lateral inhibition operating on distributed representations is a reasonable estimate of the degree of trivial or non-specific decorrelation that can be achieved simply by thresholding, or reducing the overall activity in a representation via uniform or disorganized inhibition, as has been demonstrated in other models of olfactory bulb decorrelation (e.g., Wiechert et al., 2010; see Discussion). While this may contribute to net decorrelation under some circumstances, it is markedly inferior to the specific, targeted, Mexican-hat decorrelations performed by intraglomerular local inhibition, or by lateral inhibition within appropriately topographical representations (Cleland, 2010). For example, thresholding necessarily reduces sensitivity in proportion to decorrelation, whereas on-center/inhibitory-surround mechanisms such as those studied herein can separately regulate the sensitivity and sharpness of receptive fields; e.g., nicotinic cholinergic neuromodulation 
both sharpens mitral cell receptive fields and enhances their sensitivity via concerted effects in mitral and periglomerular cells (Mandairon et al., 2006).

\section{DISCUSSION}

The circuitry of the olfactory bulb clearly serves, among other functions, to decorrelate the representations of odors with respect to their structural and perceptual similarities, as observed in the on-center/inhibitory-surround ("Mexican-hat") tuning curves of mitral cells presented with homologous series of sequentially similar odorants (Yokoi et al., 1995). Whereas different network mechanisms have been proposed to mediate this decorrelation, we demonstrate that the "classical model" of lateral inhibition cannot successfully decorrelate representations in which receptive field overlap does not reliably correlate with proximity, as is the case in the olfactory bulb. In contrast, the intraglomerular inhibition mechanism - also referred to as NTCE (Cleland and Sethupathy, 2006) - successfully decorrelates olfactory bulb representations irrespective of the physical distribution of activated glomeruli and also explains the effects of nicotinic cholinergic neuromodulation within the olfactory bulb glomerular layer (Mandairon et al., 2006). This proximity-independent decorrelation algorithm also may have application in other sensory networks in which the metric space being represented is sufficiently high-dimensional so as to require discontinuous representations in the brain, such as in piriform cortex, or the orientation columns and color blobs of area V1, or the interdigitating color, form, and depth stripes of V2 (Livingstone and Hubel, 1984; Hubel and Livingstone, 1987; Bonhoeffer and Grinvald, 1991; Roe and Ts'o, 1995). A particularly interesting example is gustatory insular cortex; while the structure of gustatory representations at this level remains unclear, the insular cortex lacks intercolumnar lateral inhibition (in direct contrast to somatosensory barrel cortex), instead exhibiting an intracolumnar variant of the feedforward intraglomerular inhibition circuit here described for the olfactory bulb (Sato et al., 2008). In general, it should not be surprising that the computational architecture of modality-selective cortical circuitry is adapted to the different properties and statistics of sensory stimuli in the relevant modalities.

\section{INVARIANCE TO CONCENTRATION}

The olfactory bulb incorporates several cell classes and circuit elements that for clarity were excluded from the present simulations. ET cells - or a subclass thereof - also receive direct afferent input from convergent OSN populations. They are resonant in the theta-band frequencies of respiration and involved in intrabulbar communication between homologous glomeruli (Hayar et al., 2004; Liu and Shipley, 2008; Zhou and Belluscio, 2008). Functionally, ET cells have been proposed to implement a global feedback normalization circuit (along with superficial short-axon cells) that maintains the mean activity level of mitral cells within a restricted functional range and predicts behavioral results to cross-concentration training (Cleland et al., 2007), although this is unlikely to be their only function. The multiple mechanisms contributing to olfactory concentration invariance have been recently reviewed (Cleland et al., 2012).

\section{OTHER BULBAR COMPUTATIONS}

Deep interneurons such as granule cells and the heterogeneous deep short-axon cells (Eyre et al., 2008, 2009) further shape the interactions among mitral cells, although they can influence odor stimulus processing only after activated mitral cells generate action potentials. Granule cells in particular form a dense synaptic network with mitral cells that comprises the external plexiform layer (EPL) of the olfactory bulb; in this layer, mitral cells excite granule cell dendrites and granule cells reciprocally inhibit the same mitral cell (recurrent inhibition) as well as other mitral cells (lateral inhibition). This lateral inhibitory network also has been proposed to decorrelate mitral cell odor representations, both in the classical topographical sense, which exhibits properties similar to the lateral inhibitory mechanism presented herein (Figures 1 B and 2C,D), and in more contemporary incarnations that recognize the absence of chemotopy in olfactory bulb (Linster et al., 2005; Arevian et al., 2008; Wick et al., 2010; Wiechert et al., 2010).

One such model has demonstrated that random lateral inhibitory projections will broadly reduce activity levels in a network, contributing to a de facto, non-specific pattern decorrelation by inhibiting all neurons such that fewer in total are active (Wiechert et al., 2010); Figure 1Aiii; also compare to the $35 \%$ decorrelation of the distributed, lateral bar in Figure 3C). Thresholded neurons accentuate this non-specific decorrelation effect by eliminating weakly activated neurons from the pattern; recurrent connections also accentuate the effect by iteratively reducing the inhibition of strongly activated neurons as the weaker neurons are inhibited out of the pattern (Wick et al., 2010). The latter effect is roughly comparable to the effects of intensity compression and normalization operations within the glomerular layer (Wachowiak et al., 2002; McGann et al., 2005; Cleland et al., 2007). However, the concomitant loss of sensitivity renders this function a poor decorrelator. A second model has proposed that lateral inhibition in this network is "activity-dependent" (Arevian et al., 2008), though the observed effect can arise simply because lateral and recurrent inhibitory effects on mitral cells summate (Egger et al., 2003, 2005); i.e., active mitral cells evoke recurrent inhibition in addition to whatever lateral inhibition they may receive, and therefore are inhibited more than are silent or less-active mitral cells. To assess the impact of this phenomenon on correlated activity, the authors measured decorrelation in a theoretical model based upon non-spiking neurons in a functionally all-to-all inhibitory network. The decorrelation observed in the model was substantially due to non-specific activity reduction owing to uniformly delivered inhibition as described above, but also exhibited an additional degree of decorrelation attributable to the selective addition of inhibition to "moderately" active mitral cells (i.e., representing recurrent inhibition). However, as this "moderate" range of mitral cell activity in bulb slice experiments extended up to a spike rate of $110 \mathrm{~Hz}$ (Figure 2 in Arevian et al., 2008), well above the normal peak rates observed in mitral cells in vivo (Rinberg et al., 2006; David et al., 2009; Chaudhury et al., 2010), it is unclear how much this additional effect would contribute to pattern decorrelation under normal conditions. In any event, granule cell-mediated lateral inhibition is unlikely to be capable of silencing mitral cells in the manner observed by Yokoi et al. (1995), as even massive lateral inhibition delivered by direct stimulation of the granule 
cell layer did not fully silence mitral cell spiking (Arevian et al., 2008), whereas periglomerular cells readily gate mitral cell activity (Gire and Schoppa, 2009).

Third, a genuine on-center/inhibitory-surround decorrelation profile can be obtained by a point-to-point map of lateral inhibitory projections in which the inhibitory weights correlate with receptive field similarities irrespective of physical location (Linster et al., 2005). Indeed, the strengths of inhibitory influences within olfactory bulb are independent of proximity (Fantana et al., 2008), though (in rats) they are also sparser than this map-based model would require. The map-based algorithm is computationally similar to lateral inhibition on a topographical representation (Figure 2C); however, it requires quantitative foreknowledge of all receptive field similarities exhibited by all pairs of glomeruli in order to weight the synapses correctly. Moreover, a given pattern of inhibitory weights of course will not be robust to the inevitable changes in receptive field similarity profiles that will occur whenever the animal moves into a new olfactory environment.

\section{LEARNING-BASED DECORRELATION IN THE EPL}

Studies of cholinergic neuromodulation within olfactory bulb make it clear that decorrelation among physically feature-similar odorants as described herein is situated primarily within the glomerular layer, and not the EPL. Specifically, blockade of nicotinic cholinergic receptors, which in olfactory bulb are found only in the glomerular layer, substantially reduces discrimination among similar odorants and broadens the receptive fields of mitral cells (Mandairon et al., 2006; Chaudhury et al., 2009), whereas blockade of muscarinic cholinergic receptors, located in the EPL, has a much smaller, possibly secondary effect. Instead, it has been proposed that EPL-based computations contribute to further, experience-dependent decorrelation based on olfactory learning rather than on the physical similarities of odorant ligands (Cecchi et al., 2001; Cleland, 2010; Cleland et al., 2012). Indeed, intrinsic learning is embedded in bulbar circuit plasticity and depends on the survival and differentiation of newly generated neurons in the deep layers of the olfactory bulb (Moreno et al., 2009; Kermen et al., 2010), as previously predicted on theoretical grounds (Cecchi et al., 2001). The progressive adaptation to real-world odor statistics that this learning theoretically enables is likely to regulate the degree of correlation among

\section{REFERENCES}

Arevian, A. C., Kapoor, V., and Urban, N. N. (2008). Activity-dependent gating of lateral inhibition in the mouse olfactory bulb. Nat. Neurosci. 11, 80-87.

Bonhoeffer, T., and Grinvald, A. (1991). Iso-orientation domains in cat visual cortex are arranged in pinwheel-like patterns. Nature 353, 429-431.

Cecchi, G. A., Petreanu, L. T., AlvarezBuylla, A., and Magnasco, M. O. (2001). Unsupervised learning and adaptation in a model of adult neurogenesis. J. Comput. Neurosci. 11, 175-182.
Chaudhury, D., Escanilla, O., and Linster, C. (2009). Bulbar acetylcholine enhances neural and perceptual odor discrimination. J. Neurosci. 29, 52-60.

Chaudhury, D., Manella, L., Arellanos, A., Escanilla, O., Cleland, T. A., and Linster, C. (2010). Olfactory bulb habituation to odor stimuli. Behav. Neurosci. 124, 490-499.

Cleland, T. A. (2010). Early transformations in odor representation. Trends Neurosci. 33, 130-139.

Cleland, T. A., Chen, S. T., Hozer, K. W., Ukatu, H. N., Wong,

related representations as a categorization process, and thereby to underlie the learning-dependence of olfactory generalization gradients (Cleland et al., 2009, 2012; Mandairon and Linster, 2009).

\section{CONCLUSION}

Both the lateral and intraglomerular inhibitory circuit elements described herein exist anatomically, whatever functions might be ascribed to them. Both are susceptible to modulation by incoming neuromodulatory fibers including cholinergic, serotonergic, and noradrenergic fibers. In the lateral inhibition model, no specific role is indicated for intraglomerular periglomerular cell inhibition of mitral cells, though it might be considered to contribute to the compression of concentration-dependent responses so as to expand the intensity tuning range (Cleland and Linster, 1999) of mitral cells. In the intraglomerular inhibition model, in turn, no specific role is mandated for periglomerular lateral axonal projections. One intriguing possibility is that they may contribute to olfactory decorrelation in a specific and limited way, e.g., by offsetting the tendency of immediately neighboring glomeruli to exhibit slightly more similar receptive fields to one another than would be predicted by chance (Soucy et al., 2009). [These particular correlations may arise owing to the recent divergence of paralogous olfactory receptor genes or epigenetic regulatory factors (Kambere and Lane, 2007), as such acquired differences appear to result in allelic or non-allelic exclusion and consequent segregation into distinct, adjacent or near-adjacent glomeruli (Serizawa et al., 2000; Ishii et al., 2001; Nguyen et al., 2007)]. While these neighborhood relationships are not sufficiently pervasive or general to underlie the entire process of decorrelation or to support a hypothesis of predictive bulbar chemotopy, the presence of anatomically localized inhibitory projections in the immediate neighborhood could facilitate decorrelation between the most reliably similar receptive fields. Such flexible deployment of cortical computational mechanisms to solve practical problems in perception is likely to be the rule, not the exception; a lateral inhibition architecture should be considered a functional adaptation to the mechanistic properties of specific modalities, and not a universal solution to all problems of decorrelation.

\section{ACKNOWLEDGMENTS}

This research was supported by NIDCD grant DC012249.

K. J., and Zheng, F. (2012) Sequential mechanisms underlying concentration invariance in biological olfaction. Front. Neuroeng. 4:21. doi:10.3389/fneng.2011.00021

Cleland, T. A., Johnson, B. A., Leon, M., and Linster, C. (2007). Relational representation in the olfactory system. Proc. Natl. Acad. Sci. U.S.A. 104 1953-1958.

Cleland, T. A., and Linster, C. (1999). Concentration tuning mediated by spare receptor capacity in olfactory sensory neurons: a theoretical study. Neural Comput. 11, 1673-1690.
Cleland, T. A., Narla, V. A., and Boudadi, K. (2009). Multiple learning parameters differentially regulate olfactory generalization. Behav. Neurosci. 123 26-35.

Cleland, T. A., and Sethupathy, P. (2006). Non-topographical contrast enhancement in the olfactory bulb. BMC Neurosci. 7, 7 . doi:10.1186/1471-2202-7-7

Cook, P. B., and McReynolds, J. S. (1998a). Lateral inhibition in the inner retina is important for spatial tuning of ganglion cells. Nat. Neurosci. 1, 714-719. 
Cook, P. B., and McReynolds, J. S. (1998b). Modulation of sustained and transient lateral inhibitory mechanisms in the mudpuppy retina during light adaptation. J. Neurophysiol. 79, 197-204.

David, F. O., Hugues, E., Cenier, T., Fourcaud-Trocme, N., and Buonviso, N. (2009). Specific entrainment of mitral cells during gamma oscillation in the rat olfactory bulb. PLoS Comput. Biol. 5, e1000551. doi:10.1371/journal.pcbi.1000551

Egger, V., Svoboda, K., and Mainen, Z. F. (2003). Mechanisms of lateral inhibition in the olfactory bulb: efficiency and modulation of spikeevoked calcium influx into granule cells. J. Neurosci. 23, 7551-7558.

Egger, V., Svoboda, K., and Mainen, Z. F. (2005). Dendrodendritic synaptic signals in olfactory bulb granule cells: local spine boost and global low-threshold spike. J. Neurosci. 25, 3521-3530.

Eyre, M. D., Antal, M., and Nusser, Z. (2008). Distinct deep short-axon cell subtypes of the main olfactory bulb provide novel intrabulbar and extrabulbar GABAergic connections. J. Neurosci. 28, 8217-8229.

Eyre, M. D., Kerti, K., and Nusser, Z. (2009). Molecular diversity of deep short-axon cells of the rat main olfactory bulb. Eur. J. Neurosci. 29, 1397-1407.

Fantana, A. L., Soucy, E. R., and Meister, M. (2008). Rat olfactory bulb mitral cells receive sparse glomerular inputs. Neuron 59, 802-814.

Gire, D. H., and Schoppa, N. E. (2009). Control of on/off glomerular signaling by a local GABAergic microcircuit in the olfactory bulb. J. Neurosci. 29, 13454-13464.

Hayar, A., Karnup, S., Shipley, M. T., and Ennis, M. (2004). Olfactory bulb glomeruli: external tufted cells intrinsically burst at theta frequency and are entrained by patterned olfactory input. J. Neurosci. 24, 1190-1199.

Hubel, D. H., and Livingstone, M. S. (1987). Segregation of form, color, and stereopsis in primate area $18 . J$. Neurosci. 7, 3378-3415.

Ishii, T., Serizawa, S., Kohda, A., Nakatani, H., Shiroishi, T., Okumura, K., Iwakura, Y., Nagawa, F., Tsuboi, A., and Sakano, H. (2001). Monoallelic expression of the odourant receptor gene and axonal projection of olfactory sensory neurones. Genes Cells 6, 71-78.

Joublin, F., Spengler, F., Wacquant, S., and Dinse, H. R. (1996). A columnar model of somatosensory reorganizational plasticity based on Hebbian and non-Hebbian learning rules. Biol. Cybern. 74, 275-286.

Kambere, M. B., and Lane, R. P. (2007). Co-regulation of a large and rapidly evolving repertoire of odorant receptor genes. BMC Neurosci. 8(Suppl. 3), S2. doi:10.1186/1471-2202-8-S3-S2

Kermen, F., Sultan, S., Sacquet, J., Mandairon, N., and Didier, A. (2010). Consolidation of an olfactory memory trace in the olfactory bulb is required for learninginduced survival of adult-born neurons and long-term memory. PLoS ONE 5, e12118. doi:10.1371/journal.pone. 0012118

Lavallee, P., and Deschenes, M. (2004). Dendroarchitecture and lateral inhibition in thalamic barreloids. J. Neurosci. 24, 6098-6105.

Linster, C., and Cleland, T. A. (2004). Configurational and elemental odor mixture perception can arise from local inhibition. J. Comput. Neurosci. $16,39-47$.

Linster, C., and Gervais, R. (1996). Investigation of the role of interneurons and their modulation by centrifugal fibers in a neural model of the olfactory bulb. J. Comput. Neurosci. 3, 225-246.

Linster, C., and Hasselmo, M. (1997). Modulation of inhibition in a model of olfactory bulb reduces overlap in the neural representation of olfactory stimuli. Behav. Brain Res. 84, 117-127.

Linster, C., Sachse, S., and Galizia, C. G. (2005). Computational modeling suggests that response properties rather than spatial position determine connectivity between olfactory glomeruli. J. Neurophysiol. 93, 3410-3417.

Liu, S., and Shipley, M. T. (2008). Multiple conductances cooperatively regulate spontaneous bursting in mouse olfactory bulb external tufted cells. J. Neurosci. 28, 1625-1639.

Livingstone, M. S., and Hubel, D. H. (1984). Anatomy and physiology of a color system in the primate visual cortex. J. Neurosci. 4, 309-356.

Mandairon, N., Ferretti, C. J., Stack, C. M., Rubin, D. B., Cleland, T. A., and Linster, C. (2006). Cholinergic modulation in the olfactory bulb influences spontaneous olfactory discrimination in adult rats. Eur. J. Neurosci. 24, 3234-3244.

Mandairon, N., and Linster, C. (2009). Odor perception and olfactory bulb plasticity in adult mammals. J. Neurophysiol. 101, 2204-2209.

McGann, J. P., Pirez, N., Gainey, M. A., Muratore, C., Elias, A.
S., and Wachowiak, M. (2005). Odorant representations are modulated by intra- but not interglomerular presynaptic inhibition of olfactory sensory neurons. Neuron 48 1039-1053.

Moreno, M. M., Linster, C., Escanilla, O. Sacquet, J., Didier, A., and Mandairon, N. (2009). Olfactory perceptual learning requires adult neurogenesis. Proc. Natl. Acad. Sci. U.S.A. 106 17980-17985.

Nguyen, M. Q., Zhou, Z., Marks, C. A., Ryba, N. J., and Belluscio, L. (2007). Prominent roles for odorant receptor coding sequences in allelic exclusion. Cell 131, 1009-1017.

Pinching, A. J., and Powell, T. P. (1971). The neuron types of the glomerular layer of the olfactory bulb. J. Cell. Sci. 9, 305-345.

Pinching, A. J., and Powell, T. P. (1972). Experimental studies on the axons intrinsic to the glomerular layer of the olfactory bulb. J. Cell. Sci. 10 637-655.

Pirez, N., and Wachowiak, M. (2008). In vivo modulation of sensory input to the olfactory bulb by tonic and activity-dependent presynaptic inhibition of receptor neurons. $J$. Neurosci. 28, 6360-6371.

Rinberg, D., Koulakov, A., and Gelperin, A. (2006). Sparse odor coding in awake behaving mice. J. Neurosci. 26 , 8857-8865.

Roe, A. W., and Ts'o, D. Y. (1995). Visual topography in primate V2: multiple representation across functional stripes. J. Neurosci. 15, 3689-3715.

Sato, H., Shimanuki, Y., Saito, M., Toyoda, H., Nokubi, T., Maeda, Y., Yamamoto, T., and Kang, Y. (2008). Differential columnar processing in local circuits of barrel and insular cortices. J. Neurosci. 28, 3076-3089.

Serizawa, S., Ishii, T., Nakatani, H., Tsuboi, A., Nagawa, F., Asano, M. Sudo, K., Sakagami, J., Sakano, H., Ijiri, T., Matsuda, Y., Suzuki, M., Yamamori, T., and Iwakura, Y. (2000). Mutually exclusive expression of odorant receptor transgenes. Nat. Neurosci. 3, 687-693.

Soucy, E. R., Albeanu, D. F., Fantana, A. L., Murthy, V. N., and Meister, M. (2009). Precision and diversity in an odor map on the olfactory bulb. Nat. Neurosci. 12, 210-220.

Suga, N., Zhang, Y., and Yan, J. (1997). Sharpening of frequency tuning by inhibition in the thalamic auditory nucleus of the mustached bat. $J$. Neurophysiol. 77, 2098-2114.

von Bekesy, G. (1967). Sensory Inhibition. Princeton: Princeton University Press.
Wachowiak, M., Cohen, L. B., and Zochowski, M. R. (2002). Distributed and concentrationinvariant spatial representations of odorants by receptor neuron input to the turtle olfactory bulb. $J$. Neurophysiol. 87, 1035-1045.

Wick, S. D., Wiechert, M. T., Friedrich, R. W., and Riecke, H. (2010). Pattern orthogonalization via channel decorrelation by adaptive networks. J. Comput. Neurosci. 28, 29-45.

Wiechert, M. T., Judkewitz, B., Riecke, H., and Friedrich, R. W. (2010). Mechanisms of pattern decorrelation by recurrent neuronal circuits. Nat. Neurosci. 13, 1003-1010.

Wiemer, J., Spengler, F., Joublin, F., Stagge, P., and Wacquant, S. (2000). Learning cortical topography from spatiotemporal stimuli. Biol. Cybern. 82, 173-187.

Yang, L., Pollak, G. D., and Resler, C. (1992). GABAergic circuits sharpen tuning curves and modify response properties in the mustache bat inferior colliculus. J. Neurophysiol. 68, 1760-1774.

Yokoi, M., Mori, K., and Nakanishi, S. (1995). Refinement of odor molecule tuning by dendrodendritic synaptic inhibition in the olfactory bulb. Proc. Natl. Acad. Sci. U.S.A. 92 3371-3375.

Zhou, Z., and Belluscio, L. (2008). Intrabulbar projecting external tufted cells mediate a timing-based mechanism that dynamically gates olfactory bulb output. J. Neurosci. 28, 9920-9928.

Conflict of Interest Statement: The authors declare that the research was conducted in the absence of any commercial or financial relationships that could be construed as a potential conflict of interest.

Received: 12 May 2011; accepted: 24 January 2012; published online: 10 February 2012.

Citation: Cleland TA and Linster C (2012) On-center/inhibitory-surround decorrelation via intraglomerular inhibition in the olfactory bulb glomerular layer. Front. Integr. Neurosci. 6:5. doi: 10.3389/fnint.2012.00005

Copyright $\odot 2012$ Cleland and Linster. This is an open-access article distributed under the terms of the Creative Commons Attribution Non Commercial License, which permits non-commercial use, distribution, and reproduction in other forums, provided the original authors and source are credited. 